\title{
Constraints on mixing angles of Majorana neutrinos
}

\author{
Rathin Adhikari* and G. Rajasekaran ${ }^{\dagger}$ \\ The Institute of Mathematical Sciences, C.I.T Campus, Taramani, Chennai-600 113, India
}

\begin{abstract}
By combining the inputs from the neutrinoless double beta decay and the fits of cosmological models of dark matter with solar and atmospheric neutrino data, we obtain constraints on two of the mixing angles of Majorana neutrinos, which become stronger when coupled with the reactor neutrino data. These constraints are strong enough to rule out Majorana neutrinos if the small angle solution of solar neutrino puzzle is borne out.
\end{abstract}

PACS number(s): 14.60.Pq, 14.60.St, 23.40.Bw, 26.65.+t

*rathin@imsc.ernet.in

†graj@imsc.ernet.in 
It is well known [1,2] that unless neutrinos are very massive and nonrelativistic, or interact through both left and right-handed currents, experimental data on neutrino-induced reactions cannot distinguish between Dirac and Majorana neutrinos. Neutrinoless double beta decay remains as the only feasible tool to probe this question. Although experiments [3] 5] have so far provided only upper limits on the rate of this decay, recent limits [5] combined with other inputs on neutrino physics might already lead to important information on whether neutrinos are Dirac or Majorana particles. These other inputs are indications from the analysis of the cosmic microwave background for the presence of a hot dark matter component which is presumably neutrinos of mass $\approx 1 \mathrm{eV}[6,8,9]$ and the indications [10 12 from the analysis of solar and atmospheric neutrinos that neutrinos do oscillate and that the mass differences among the three neutrinos are much smaller than this scale of $1 \mathrm{eV}$.

We show in this note that if neutrinos are Majorana particles, a combined study of all the above pieces of data leads to rather stringent restrictions on two of the mixing angles that occur for three flavors of neutrinos. If these results are then confronted with the values of these mixing angles allowed by solar, atmospheric and reactor neutrino data, the allowed regions are further narrowed and in fact, in a few cases, one is already close to contradiction, thus leading to the conclusion that neutrinos are not Majorana particles.

In the three flavor mixing scheme the neutrino flavor eigenstates $\nu_{\alpha}=\nu_{e, \mu, \tau}$ are related to the mass eigenstates $\nu_{i}=\nu_{1,2,3}$ by

$$
\nu_{\alpha}=\sum_{i} U_{\alpha i} \nu_{i}
$$

where $U_{\alpha i}$ are the elements of the unitary mixing matrix $U$. We note that for Majorana neutrino [2,13] there are three $C P$ violating phases in contrast to the case of Dirac neutrino which has only one phase. We use the parametrization [13]:

$$
U=\left(\begin{array}{ccc}
c_{\omega} c_{\phi} & s_{\omega} c_{\phi} e^{-i \delta_{1}} & s_{\phi} e^{-i \delta_{2}} \\
-s_{\omega} c_{\psi} e^{i \delta_{1}}-c_{\omega} s_{\psi} s_{\phi} e^{i\left(\delta_{2}+\delta_{3}\right)} & c_{\omega} c_{\psi}-s_{\omega} s_{\psi} s_{\phi} e^{i\left(\delta_{3}+\delta_{2}-\delta_{1}\right)} & s_{\psi} c_{\phi} e^{i \delta_{3}} \\
s_{\omega} s_{\psi} e^{i\left(\delta_{1}-\delta_{3}\right)}-c_{\omega} c_{\psi} s_{\phi} e^{i \delta_{2}} & -c_{\omega} s_{\psi} e^{-i \delta_{3}}-s_{\omega} c_{\psi} s_{\phi} e^{i\left(\delta_{2}-\delta_{1}\right)} & c_{\psi} c_{\phi}
\end{array}\right)
$$


where $\delta_{1}, \delta_{2}$ and $\delta_{3}$ are the three $C P$-violating phases and $c$ and $s$ stand for sine and cosine of the associated mixing angle $\omega, \phi$ or $\psi$ placed as subscript.

The rate for the neutrinoless double beta decay depends on the following combination of the neutrino parameters [2]:

$$
m_{0 \nu \beta \beta}=\left|\sum_{i=1,2,3} \eta_{i} U_{e i}^{2} m_{\nu_{i}}\right|
$$

where $m_{\nu_{i}}$ are the Majorana neutrino masses, $U_{e i}$ the elements of the first row of the mixing matrix given in equation $(2), \eta_{i}=(1 / i) \eta_{i}^{C P}= \pm 1$ and $\eta_{i}^{C P}$ is the $C P$ parity of Majorana neutrino $\nu_{i}$. Although neutrinoless double beta decay has not yet been seen experimentally, the experimental upper limits on this rate have recently improved to a significant extent. In particular one may refer to the results of the Tellurium [3] and Germanium experiments [4.5]. The strongest upper limit so far comes from the Germanium experiment [5, 14] and it is

$$
\begin{aligned}
m_{0 \nu \beta \beta} & <0.56 \mathrm{eV} \quad(99 \% \text { confidence level }) \\
& <0.46 \mathrm{eV} \quad(90 \% \text { confidence level })
\end{aligned}
$$

These numbers have been obtained using the nuclear matrix elements calculated in [15]. We shall take into account the uncertainties in this calculation (see below). Using the conservative number $0.56 \mathrm{eV}$ we have

$$
\left|\sum_{i=1,2,3} \eta_{i} U_{e i}{ }^{2} m_{\nu_{i}}\right|<0.56 \mathrm{eV}
$$

Next we consider the fits to the recent data on the anisotropies of the cosmic microwave background radiation [16] and the large scale structure of the universe [17]. Best fit [9,8] requires a mixture of $10 \%$ ordinary baryonic matter, $70 \%$ cold and $20 \%$ hot dark matter with $\Omega_{m}=1$. If the hot component is identified with neutrinos, the model implies [6]

$$
\sum_{i=1,2,3} m_{\nu_{i}} \approx 5 \mathrm{eV}
$$

Right hand side of (6) is not expected to be less than $3 \mathrm{eV}$ for $\Omega_{m}=1$ [7] as otherwise there is too much small scale power [18]. On the other hand, solar and atmospheric neutrino 
data suggest that the two mass-squared differences among the three neutrinos are very small [10 12]: $m_{2}^{2}-m_{1}^{2} \approx 10^{-5} \mathrm{eV}^{2}$ or smaller and $m_{3}^{2}-m_{1}^{2} \approx 10^{-3}-10^{-2} \mathrm{eV}^{2}$. Hence we take all three neutrinos as almost degenerate in mass and using (6),

$$
m_{\nu_{i}} \approx m_{\nu} \approx 1.7 \mathrm{eV}
$$

We shall allow $m_{\nu}$ to vary over a range around $1.7 \mathrm{eV}$. This will take care of the uncertainties of cosmological models as well as those of the calculations of the nuclear matrix elements in double beta decay, since only the ratio $0.56 / m_{\nu}$ enters into our analysis. Any possible improvement in the neutrinoless double beta decay limit can also be incorporated by scaling $m_{\nu}$ appropriately.

Combining all the inputs, we have the basic inequality

$$
\left|\left(\eta_{1} \cos ^{2} \omega+\eta_{2} \sin ^{2} \omega e^{-i 2 \delta_{1}}\right) \cos ^{2} \phi+\eta_{3} \sin ^{2} \phi e^{-i 2 \delta_{2}}\right|<\frac{0.56}{m_{\nu}}
$$

where $m_{\nu}$ is expressed in $\mathrm{eV}$. One can rewrite this inequality in terms of two effective phases by combining $\eta_{i}$ and $\delta_{i}$. However, to make our discussion on $C P$ conserving and $C P$ violating cases more transparent we have kept both $\eta_{i}$ and $\delta_{i}$ above. We proceed to extract the bounds on the mixing angles $\omega$ and $\phi$ implied by this inequality for various choices of $\eta_{i}$ , $\delta_{i}$ and typical values of $m_{\nu}$ favored by the cosmological models. It is to be noted that in contrast to the usual oscillation phenomena studied in neutrino physics, $C P$ violation plays an important role in neutrinoless double beta decay.

We shall first consider $\delta_{1}=\delta_{2}=0$ in (8). Out of eight possible combinations for different values of $\eta_{i}$ in (8), four combinations are equivalent to the other four, as only the overall magnitude in the left hand side of this inequality is constrained. So we shall analyse (8) on the basis of four cases : Case I $: \eta_{1}=\eta_{2}=\eta_{3}= \pm 1$; Case II $: \eta_{1}=-\eta_{2}=\eta_{3}= \pm 1$; Case III $:-\eta_{1}=\eta_{2}=\eta_{3}= \pm 1$ and Case IV: $\eta_{1}=\eta_{2}=-\eta_{3}= \pm 1$. Case I is the natural choice for $\eta_{i}$, if there exists a symmetry linking the three generations. But then the left hand side of (8) is unity and so unless $m_{\nu} \leq 0.56 \mathrm{eV}$, the inequality cannot be satisfied [20]. Since such low values of $m_{\nu}$ are not expected in the cosmological models, we conclude that the case 
of equal intrinsic $C P$ parities for the three Majorana neutrinos is not favored. The allowed regions in $\omega$ and $\phi$ for cases II, III and IV are plotted in figures 1, 2 and 3 respectively for $m_{\nu}=1.7 \mathrm{eV}$. For both II and III, small $\phi(\phi \approx 0)$ requires $\omega$ to be in the region of $45^{\circ}$. Whereas small $\omega$ in II is not allowed at all, small $\omega$ in III requires large $\phi$. Case IV leads to a constraint condition on $\phi$, that is independent of $\omega$ and requires $\phi$ to be in the region of $45^{\circ}$. Figures 4 and 5 show the total allowed regions for all possible combinations of $\eta_{i}$ (i.e., all the cases I, II, III and IV) for $m_{\nu}=6 \mathrm{eV}$ and $0.64 \mathrm{eV}$ respectively. One may note that for $m_{0 \nu \beta \beta} / m_{\nu} \rightarrow 0$, the allowed values of $\omega$ and $\phi$ are constrained to lie on the three curves $: \tan ^{2} \phi= \pm \cos 2 \omega, 1$. Our figure 4 (where $m_{0 \nu \beta \beta} / m_{\nu}=0.093$ ) gives a small width to these curves.

We next consider $C P$ violating case. Now, the choice $\eta_{1}=\eta_{2}=\eta_{3}= \pm 1$, is not ruled out. Thus $C P$ violation is capable of changing the conclusions dramatically. Figures 6-8 show the allowed regions in $\omega$ and $\phi$ for a few choices of the parameters $\delta_{1}$ and $\delta_{2}$.

We have not considered "maximal" $C P$ violation $\delta_{1}=\pi / 2$ and $/$ or $\delta_{2}=\pi / 2$ since these cases are subsumed by the choices of relative negative $\eta_{i}$, as far as our inequality (8) is concerned. Therefore, it is important to keep in mind that our figures (1-5) are relevant for such "maximal" $C P$-violating cases also. In particular, figure 1 and figure 2 can be considered to be the "maximally" $C P$-violating case of $\delta_{1}=\pi / 2$ and $\delta_{2}=0$.

Let us now compare the above bounds on the mixing angles with the results of the analysis of data from reactor, solar and atmospheric neutrino experiments. Before we make this comparison, we must comment on the role of $C P$ violation in these experiments. We note that even for Majorana neutrinos, the oscillation phenomena are controlled by a single $C P$-violating phase $\delta$ [21].

The CHOOZ reactor experiment [22] interpreted within a three flavor framework 23] leads to the constraint

$$
\phi \leq 12.5^{\circ}
$$

Although $C P$ violation was neglected in reference [23], it is easy to see that (9) is valid 
even if $C P$ is violated. Comparing (9) with figure 3 , we see that case IV is ruled out, while comparisons with figures 1 and 2 rule out all values of $\omega$ except those in the region of $45^{\circ}$. These are for $m_{\nu}=1.7 \mathrm{eV}$. The results are weakened for $m_{\nu}=0.64 \mathrm{eV}$ (figure 5) and are further strengthened for $m_{\nu}=6 \mathrm{eV}$ (figure 4). If $C P$ is violated, with $\delta_{1}=0$ and $\delta_{2} \neq 0$ (figure 6), the above conclusion is still valid. But for $m_{\nu}=1.7 \mathrm{eV}, \pi / 8 \gtrsim \delta_{1} \lesssim 3 \pi / 8$ and $\delta_{2}=0$, ( figure 7 ) as well as $\pi / 8 \gtrsim \delta_{1}, \delta_{2} \lesssim 3 \pi / 8$ (figure 8 ) there is no allowed region at all after using (9).

It is important to note that as a consequence of (9), the effect of $C P$ violation in all the neutrino-oscillation phenomena is much reduced since the $C P$ violating phase factors $e^{ \pm i \delta}$ always occur in combination with $\sin \phi$, as $\sin \phi e^{ \pm i \delta}$. Hence it is legitimate to compare with the results of analyses of solar and atmospheric neutrinos even though $C P$ violation is usually ignored in those analyses.

Analysis of solar neutrino data on the basis of the MSW effect [24] leads to the allowed $\omega$ values of either $2^{\circ}-3^{\circ}$ or $20^{\circ}-40^{\circ}$ for $\phi \leq 12.5^{\circ}$. So comparing with the above result, we conclude that the result of the present analysis contradicts the small $\omega$ MSW solution $\left(\omega \approx 2^{\circ}-3^{\circ}\right)$, but there exists some overlap with the large $\omega$ MSW solution $\left(\omega \approx 20^{\circ}-40^{\circ}\right)$. Also there is no contradiction with the vaccuum oscillation as a solution of the solar neutrino problem since this also requires large $\omega$ [27]. Finally there is no contradiction with the results of the atmospheric neutrino analysis [24] since this does not involve $\omega$.

So far we have used the information on $m_{\nu}$ from cosmology to get results on the mixing angles which were then compared with the results of reactor, solar and atmospheric neutrinos. In view of the uncertainties of cosmological models, one can ask what kind of information on the quasi-degenerate mass for Majorana neutrinos can be obtained from our analysis, if we drop the cosmological input completely. It is clear from our figure 5, that for the small $\omega$ MSW solution of the solar neutrino problem, we get an upper bound on $m_{\nu}$ of about $0.7 \mathrm{eV}$ and we have checked that this upper bound becomes $4 \mathrm{eV}$ for the large $\omega$ MSW solution.

The quantitative results of our analysis are contained in figures 1-8 in the form of re- 
strictions in $\omega$ and $\phi$. We may also state two qualitative conclusions that emerge from our analysis.

1. If neutrinos are Majorana fermions and $C P$ is conserved, all the three neutrinos cannot have same $C P$ parities. This conclusion (which perhaps is well-known and is included here only for the sake of completeness) may be important for model building.

2. If neutrinos are Majorana fermions, the mixing angle $\omega$ cannot be small. Hence, if the small $\omega$ solution turns out to be the only correct solution of the solar neutrino problem, then neutrinos cannot be Majorana fermions. This will have serious consequences for models intended to explain small neutrino masses.

The above results and conclusions are based on the present indications that the experiments on the neutrinoless double beta decay require $m_{0 \nu \beta \beta}$ to be less than a fraction of an $\mathrm{eV}$ while models with neutrinos as the hot component of dark matter require $m_{\nu}$ to be higher than about $1 \mathrm{eV}$. The restrictions become more severe and the conclusions become stronger if the upper limit on $m_{0 \nu \beta \beta}$ decreases [26] and/or $m_{\nu}$ increases.

After the first submission of our manuscript, we came across the papers of Georgi and Glashow 28] and of Branco, Rebelo and Silva-Marcos [29 whose contents have partial overlap with our work. Our analysis is more general than both these works in which $\phi$ is put zero and further we have considered the $C P$-violating cases in detail.

\section{Acknowledgment}

We thank Raj Gandhi, Mohan Narayan and S. Uma Sankar for discussions and Sandip Pakvasa for a useful communication. We thank Rahul Sinha for an important discussion that clarified the role of $C P$ violation in neutrino oscillations. 


\section{REFERENCES}

[1] B. Kayser, R. Shrock, Phys. Lett. B 112, 137 (1982).

[2] R. N. Mohapatra and P. B. Pal, Massive neutrinos in Physics and Astrophysics, World Scientific, (1991); B. Kayser et al, The Physics of massive neutrinos, World Scientific, (1989).

[3] T. Bernatowich et al, Phys. Rev. Lett. 69, 2341 (1992); Phys. Rev. C 47, 806 (1993).

[4] A. Balysh et al (Heidelberg- Moscow Experiment), Phys. Lett. B 356, 450 (1995).

[5] M. Günther et al, Phys. Rev. D 55, 54 (1997); L. Baudis et al (Heidelberg- Moscow Experiment), Phys. Lett. B 407, 219 (1997).

[6] J. R. Primack, Phys. Rev. Lett, 74, 2160 (1995).

[7] J. R. Primack, Private Communication.

[8] E. Gawiser and J. Silk, Science, 280, 1405 (1998); J. R. Primack, Science, 280, 1398 (1998).

[9] J. R. Primack, Dark matter and Structure Formation, Presented in the Jerusalem Winter School 1996. astro-ph/9707285; J. R. Primack and M. A. K. Gross astro-ph/9810204.

[10] B. T. Cleveland et al, Nucl. Phys. B38 (Proc. Suppl.) (1995) 47; Y. Fukuda et al, Phys. Rev. Lett. 77 , $1683(1996)$

[11] Y. Fukuda et al, hep-ex/9803006, 9805006, 9805021 and 9807003.

[12] N. Hata and P. Langacker, Phys. Rev. D 56, 6107 (1997); P. Langacker, hepph/9811460.

[13] K. Zuber, Phys. Rep. 305, 295 (1998); we have corrected some printing errors in the mixing matrix given in this report.

[14] H. Päs, Private communication. 
[15] K. Muto, A. Staudt and H. V. Klapdor-Kleingrothaus, Europhys. Lett. 13, 31 (1990).

[16] K. M. Gorski et al, Astrophys. J. Lett. 430, L89 (1994).

[17] G. Efstathiou, J. R. Bond, S. D. M. White, Mon. Not. Roy. Astron. Soc. 258, P1 (1992).

[18] Although the observations of high redshift supernovae have been interpreted [19] in terms of a nonvanishing cosmological constant $\Lambda$, cosmological models with $\Omega_{m}+\Omega_{\Lambda}=1$ do not provide good fit to the structure data (CMBR anisotropies and the large-scale structure). Leaving this puzzle to be resolved by the cosmologists, we shall stick to equation (6) and deduce the consequences for neutrino physics.

[19] S. Perlmutter et al, Nature 391, 51 (1998); P. M. Garnavich et al, ApJ 493, L53 (1998); A. G. Riess et al, astro-ph/9805201; B. P. Schmidt et al, astro-ph/9805200.

[20] Although specific value for $m_{\nu}$ is mentioned here as well as below, it must be scaled suitably if the calculated value of the nuclear matrix element in neutrinoless double beta decay changes or if the experimental limit on this decay improves, since only the ratio of the upper limit of $m_{0 \nu \beta \beta}$ to $m_{\nu}$ enters into our analysis.

[21] M. Doi et al, Phys. Lett. B 102 , 323 (1981); S. M. Bilenky et al, Phys. Lett. B 94, 495 (1980).

[22] The CHOOZ Collaboration, M. Appolonio et al , Phys. Lett. B 420, 397 (1998)

[23] Mohan Narayan, G. Rajasekaran and S. Uma Sankar, Phys. Rev. D 58, 031301 (1998).

[24] M. Narayan et al, Phys. Rev. D 53, 2809 (1996); G. L. Fogli et al, Phys. Rev. D 54, 2048 (1996); M. Narayan et al, Phys. Rev. D 56, 437 (1997) ; G. L. Fogli et al, Phys. Lett. B 434, 333 (1998).

[25] L. Baudis et al, hep-ex/9902014.

[26] In fact this has already happened; according to reference [25], $m_{0 \nu \beta \beta}<0.2 \mathrm{eV}$ at $90 \%$ confidence level. 
[27] S. L. Glashow et al, Phys. Lett. B 190, 199 (1987); B. Faid et al, Phys. Rev. D 55, 1353 (1997); P. Osland et al, hep-ph/9806339; S. L. Glashow et al, hep-ph/9808470.

[28] H. Georgi and S. L. Glashow, hep-ph/9808293.

[29] G. C. Branco et al, hep-ph/9810328. 


\section{FIGURES}

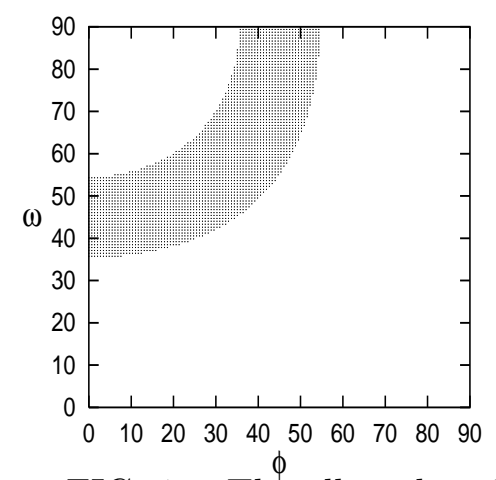

FIG. 1. The allowed region in $(\omega, \phi)$ is shown shaded for $m_{\nu}=1.7 \mathrm{eV}, \eta_{1}=-\eta_{2}=\eta_{3}= \pm 1$ and $\delta_{1}=\delta_{2}=0$.

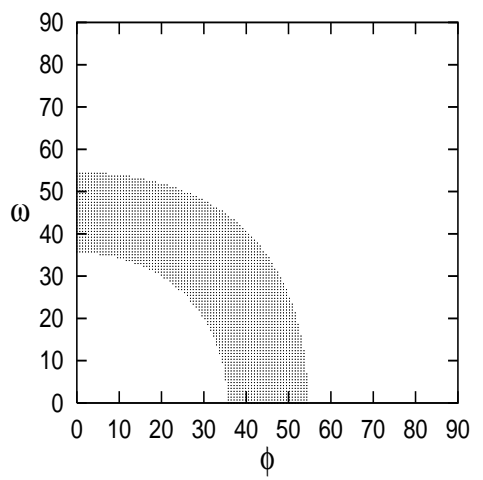

FIG. 2. Same as figure 1 but $-\eta_{1}=\eta_{2}=\eta_{3}= \pm 1$.

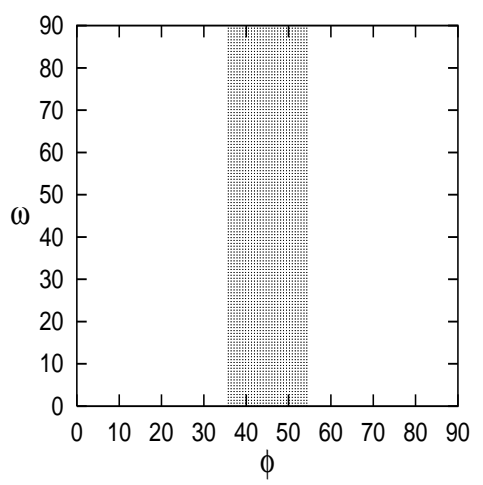

FIG. 3. Same as figure 1 but $\eta_{1}=\eta_{2}=-\eta_{3}= \pm 1$. 


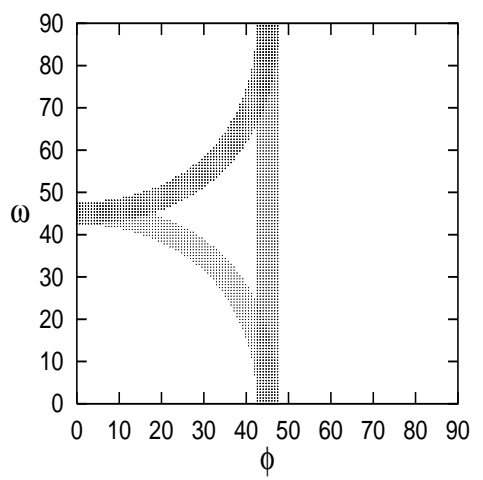

FIG. 4. Allowed region for all possible combinations of $\eta_{i}, m_{\nu}=6 \mathrm{eV}$, and $\delta_{1}=\delta_{2}=0$.

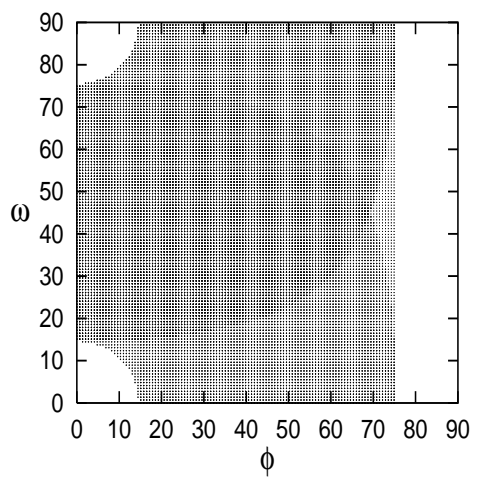

FIG. 5. Same as figure 4 but for $m_{\nu}=0.64 \mathrm{eV}$.

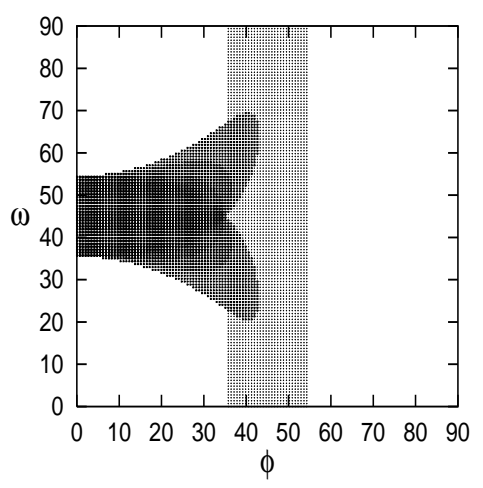

FIG. 6. Allowed region for all possible $\eta_{i}, m_{\nu}=1.7 \mathrm{eV}$ and $\delta_{1}=0, \pi / 8 \leq \delta_{2} \leq 3 \pi / 8$. 


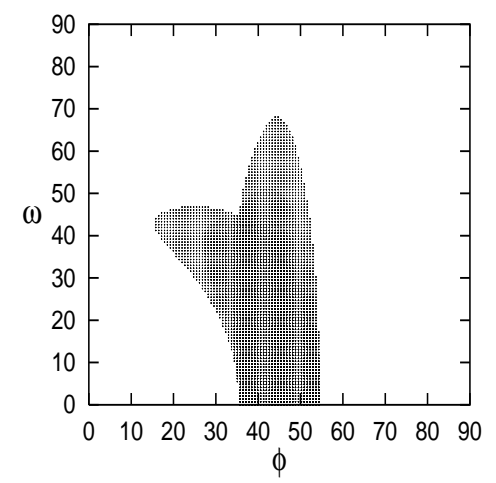

FIG. 7. Allowed region for all possible $\eta_{i}, m_{\nu}=1.7 \mathrm{eV}$ and $\pi / 8 \leq \delta_{1} \leq 3 \pi / 8, \delta_{2}=0$.

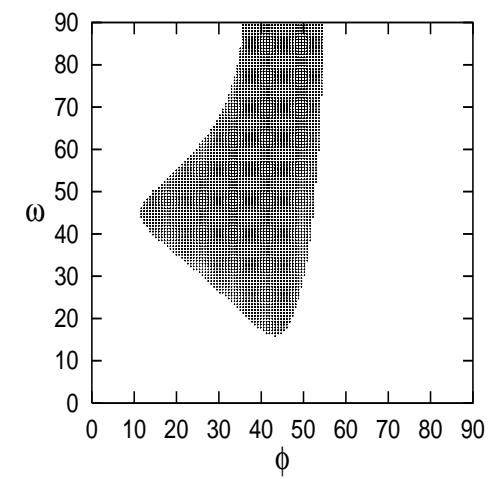

FIG. 8. Allowed region for all possible $\eta_{i}, m_{\nu}=1.7 \mathrm{eV}$ and $\pi / 8 \leq \delta_{1}, \delta_{2} \leq 3 \pi / 8$. 